$\Phi=-$

\title{
LRS Bianchi type-I Universe with anisotropic dark energy and special form of deceleration parameter in $f(\mathrm{R}, \mathrm{T})$ gravity
}

\author{
R.P.Wankhade ${ }^{1 *}$, A.S. Bansod ${ }^{2}$ \\ ${ }^{1}$ Jawaharlal Darda Institute of Engineering \& Technology, Yavatmal (445001) INDIA \\ ${ }^{2}$ Department of Mathematics, V.Y.W.S, Badnera Polytechnic, Badnera, INDIA \\ *Corresponding author E-mail:r_wan@ rediffmail.com
}

\begin{abstract}
In this paper, LRS Bianchi type-I space-time is considered in the presence of perfect fluid source in the frame work of $f(R, T)$ gravity (Harko et al. in Phys.Rev. D 84:024020, 2011) where $f(R, T)$ is an arbitrary function of Ricci scalar $R$ and trace of the energy momentum tensor $T$. The Einstein's field equations have been solved by taking into account the special form of deceleration parameter (Singha A., Debnath U.: Int.J. Theor. Phys.48, 2009). We observe that in $f(\mathrm{R}, \mathrm{T})$ gravity, an extra acceleration is always present due to coupling between matter and geometry. The geometrical and physical aspect of the model is also studied.
\end{abstract}

Keywords:LRS Bianchi Type-I Space-Time; Special Form of Deceleration Parameter; $f(R, T)$ Gravity.

\section{Introduction}

The observational data of high redshift type SN Ia supernovae [12] shows that our Universe for later stages of evolution indicates accelerated expansion and confirmed later by cross checks from the cosmic microwave background radiation and large scale structure [3-6] strongly suggest that the Universe is spatially flat and dominated by an exotic component with large negative pressure, referred to as dark energy [7-10].

In order to explain the nature of dark energy and the accelerated expansion, various theoretical models have been proposed such as quintessence, phantom energy, k-essence, tachyon, f-essence, chaplygin gas, etc. Among these different models of DE, the modified gravity theories are $f(R)$ gravity [11-15], Gauss-Bonnet gravity or $f(G)$ gravity [16-20] and $f(T)$ gravity [21-23], where $T$ is scalar torsion. These modified gravities have recently been verified to explain the late-time accelerated expansion of the Universe.

Recently, Harko et al. [24] proposed $f(R, T)$ modified theories of gravity, where the gravitational Lagrangian is the function of the Ricci scalar $R$ and the trace of the stress energy tensor $T$. The $f(R, T)$ gravity model depends on a source term, representing the variation of the matter stress energy tensor with respect to the metric. A general expression for this source term is obtained as a function of the matter Lagrangian $L_{m}$ so that each choice of $L_{m}$ would generate a specific set of field equations. The $f(R, T)$ gravity models can explain the late time cosmic accelerated expansion of the Universe.

A Bianchi Type-I cosmological model, being the generalization of flat Friedmann-Robertson-Walker (FRW) model, is one of the simplest models of the anisotropic universe. Therefore, we confine ourselves to Bianchi type-I model in the context of $f(R, T)$ gravity.
By choosing a particular form of the deceleration parameter, which gives an early deceleration and late time acceleration for dust dominated model, [30] shows that this sign flip which can be obtained by a simple trigonometric potential. The quintessence model [31] with a minimally coupled scalar field by taking a special form of decelerating parameter $q$ in such a way that which provides an early deceleration and late time acceleration for borotropic fluid and Chaplygin gas dominated models. Motivated from the studies outlined above we choose a form of $q$ as a function of the scale factor ' $a$ 'so that it has the desired property of a signature flip.

In the present paper, LRS Bianchi type-I cosmological model in the context of $f(R, T)$ gravity theory have been studied. The solutions of the Einstein's field equations have been obtained by applying special form of deceleration parameter. Some features of the evolution of the metric and the dynamics of the anisotropic DE fluid have been examined.

\section{Gravitational field equations of $f(R, T)$ gravity}

The action for the modified $f(R, T)$ gravity is

$S=\frac{1}{16} \int f(R, T) \sqrt{-g} d^{4} x+\int L_{m} \sqrt{-g} d^{4} x$,

where $R, T$ and $L_{m}$ are the Ricci scalar, the trace of stress-energy tensor of matter and the matter Lagrangian density respectively. We define the stress-energy tensor of matter as

$T_{i j}=-\frac{2}{\sqrt{-g}} \frac{\delta \sqrt{-g} L_{m}}{\delta g^{i j}}$.

The gravitational field equation of $f(R, T)$ gravity is given by 


$$
\begin{aligned}
& f_{R}(R, T) R_{i j}-\frac{1}{2} f(R, T)+\left(g_{i j} \nabla^{i} \nabla_{i}-\nabla_{i} \nabla_{i}\right) f_{R}(R, T), \\
& =8 \pi T_{i j}-f_{T}(R, T) T_{i j}-f_{T}(R, T) \Theta_{i j} \\
& \text { where } \\
& f_{R}(R, T)=\frac{\partial f(R, T)}{\partial R}, f_{T}=\frac{\partial f(R, T)}{\partial T}, \Theta_{i j}=-2 T_{i j}-p g_{i j}
\end{aligned}
$$

and $\nabla_{i}$ denote the covariant derivative.

The stress-energy tensor is given by

$$
T_{i j}=T_{i j}^{(m)}+T_{i j}^{(d e)} \text {, }
$$

Where $T_{j}^{(m) i}$ and $T_{j}^{(d e) i}$ are energy momentum tensor of perfect fluid and dark energy respectively. These are given by $T_{j}^{(m) i}=\operatorname{diag}\left[-\rho^{(m)}, p^{(m)}, p^{(m)}, p^{(m)}\right]$,

and

$$
\begin{aligned}
T_{j}^{(d e) i} & =\operatorname{diag}\left[-\rho^{(d e)}, p_{x}{ }^{(d e)}, p_{y}{ }^{(d e)}, p_{z}{ }^{(d e)}\right] \\
& =\operatorname{diag}\left[-1, \omega^{(d e)} \rho^{(d e)}, \omega^{(d e)} \rho^{(d e)}, \omega^{(d e)} \rho^{(d e)}\right]
\end{aligned}
$$

where $p^{(m)}$ and $\rho^{(m)}$ are the pressure and energy density of perfect fluid components whereas $\rho^{(d e)}$ represent the energy density of dark energy components.

In order to describe the early universe, here we assumed the string fluid as source of matter.

The field equations in $f(R, T)$ gravity also depend on the physical nature of the matter field through the tensor $\Theta_{i j}$. Hence several theoretical models can be obtained for different choice of $f(R, T)$ The cosmological consequences for the class $f(R, T)=R+2 f(T)$ have been recently discussed in details by many authors [25-30]. Adhav [25] has presented spatially homogeneous and anisotropic Bianchi type-I model in $f(R, T)$ gravity while Reddy et al, [2627] have studied Bianchi type-III and Kaluza-Klein cosmological models in this theory. Chaubey and Sukla [28], Samanta [29], have studied cosmological models in $f(R, T)$ gravity in different Bianchi type space-times.

Recently, Shamir et al. [33] and Chaubey et al. [28] have discussed Bianchi type-I \& V and a general class of Bianchi models respectively in $f(R, T)$ gravity by considering $f(R, T)=R+2 f(T)$.

In this paper we assume a particular form of the functions $f_{1}(R)=\lambda R$ and $f_{2}(T)=\lambda T$ where $\lambda$ is arbitrary parameters.

Now using $c=G=1$, equation (3) reduces to

$R_{i j}-\frac{1}{2} R g_{i j}=\left(\frac{8 \pi+\lambda}{\lambda}\right) T_{i j}+\left(p+\frac{1}{2} T\right) g_{i j}$.

The physical interpretation of the additional term $\left(p+\frac{1}{2} T\right)$ so obtained in the above equation has been given by [34-35] and they proposed that $T=\rho-3 p=(1-3 \gamma) \rho$, where $p$ is the isotropic pressure and $T$ is the trace of energy-momentum tensor and $0 \leq \gamma \leq 1$ is the equation of state parameter.

\section{Metric and field equations}

The LRS Bianchi-Type-I line element can be written as $d s^{2}=d t^{2}-A(t)^{2} d x^{2}-B(t)^{2}\left(d y^{2}+d z^{2}\right)$,

where $A(t)$ and $B(t)$ are the scale factors (metric potential) and functions of the cosmic time $t$ only (non-static case).

The Einstein's field equations (7) for metric (8) can be written as

$$
\begin{aligned}
& \left(\frac{\dot{B}}{B}\right)^{2}+2 \frac{\ddot{B}}{B}=-\left(\frac{8 \pi+\lambda}{\lambda}\right)\left(p^{(m)}+\omega^{(d e)} \rho^{(d e)}\right)-\frac{1}{2}\left(\rho^{(m)}-p^{(m)}\right), \\
& \frac{\ddot{A}}{A}+\frac{\ddot{B}}{B}+\frac{\dot{A} \dot{B}}{A B}=-\left(\frac{8 \pi+\lambda}{\lambda}\right)\left(p^{(m)}+\omega^{(d e)} \rho^{(d e)}\right)-\frac{1}{2}\left(\rho^{(m)}-p^{(m)}\right), \\
& \left(\frac{\dot{B}}{B}\right)^{2}+2 \frac{\dot{A} \dot{B}}{A B}=\left(\frac{8 \pi+\lambda}{\lambda}\right)\left(p^{(m)}+\rho^{(d e)}\right)+\frac{1}{2}\left(\rho^{(m)}-p^{(m)}\right),
\end{aligned}
$$

where dot $(\cdot)$ indicates the derivative with respect to $t$

The mean Hubble parameter $H$ for LRS Bianchi type-I metric may given by

$H=\frac{\dot{a}}{a}=\frac{1}{3}\left(\frac{\dot{A}}{A}+2 \frac{\dot{B}}{B}\right)$.

The directional Hubble parameters in the direction $x, y$ and $z$ respectively can be define as

$H_{x}=\frac{\dot{A}}{A}$ and $H_{y}=H_{z}=\frac{\dot{B}}{B}$.

The anisotropy parameter of the expansion is define as

$\Delta=\frac{1}{3} \sum_{i=1}^{3}\left(\frac{H_{i}-H}{H}\right)^{2}$,

where $H_{i}(i=1,2,3)$ represent the directional Hubble parameters in the directions of $x, y$ and $z$ axis respectively.

The shear scalar $\sigma^{2}$, defined by

$\sigma^{2}=\frac{3}{2} \Delta H^{2}$.

Here we consider that the universe is dominated by dark energy components and these dark energy components interact minimally with perfect fluid. Thus, the energy momentum tensor for perfect fluid and dark energy sources may conserve separately. The energy conservation equations for perfect fluid and dark energy components are respectively given by

$\dot{\rho}^{(m)}+3\left(\rho^{(m)}+p^{(m)}\right) H+\frac{\left(1+\omega^{(m)}\right)}{2} \dot{\rho}^{(m)}=0$.

$\dot{\rho}^{(d e)}+3\left(\omega^{(d e)}+1\right) \rho^{(d e)} H=0$.

The EoS parameter of perfect fluid is constant i.e. $\omega^{(m)}=\frac{p^{(m)}}{\rho^{(m)}}=$ cons $\tan t$ although the current cosmological data from

SNIa, CMB and large scale structures mildly favour the time dependent EoS parameter for dark energy component. Hence equation (16) reduces to

$\left(\frac{3-\omega^{(m)}}{2}\right) \dot{\rho}^{(m)}+3\left(\omega^{(m)}+1\right) \rho^{(m)} H=0$.

In order to solve the system completely we impose a special form of deceleration parameter as

$q=-\frac{\ddot{a} a}{\dot{a}^{2}}=-1+\frac{\alpha}{1+a^{\alpha}}$,

where $a$ is mean scale factor of the universe, $\alpha(>0)$ is constant. This law has been recently proposed by Singha and Debnath [32] for FRW metric. From figure (1) we have seen that $q$ decreases from +1 to -1 for evolution of the universe.

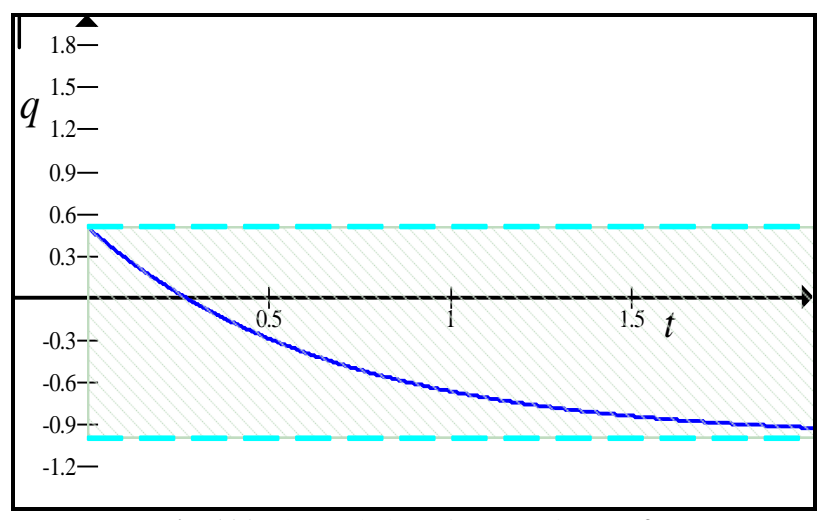

Fig. 1(a): The Variation of $q$ vs. $t$ for $\alpha=2$. 


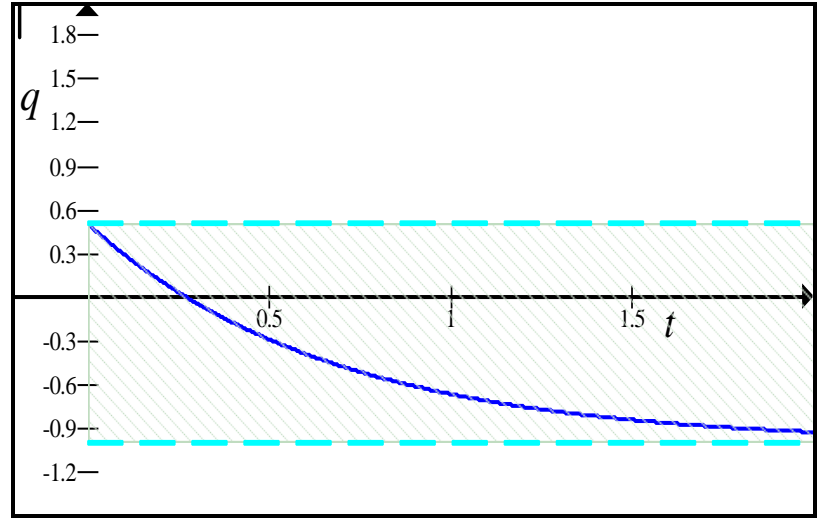

Fig. 1(b): The Variation of $q$ vs. $t$ for $\alpha=3 / 2$.

From (19) after integrating, we obtain the Hubble parameter as $H=\frac{\dot{a}}{a}=m\left(1+a^{-\alpha}\right)$,

where $\mathrm{m}$ is an arbitrary constant of integration.

Here we assume the deceleration parameter as given in (19), which can be integrated twice to give $H=\frac{\dot{a}}{a}$ as in equation (20) and the average scale factor as

$a=\left(e^{m \alpha t}-1\right)^{\frac{1}{\alpha}}$,

The spatial volume is given by

$V=a^{3}=A B^{2}$.

i.e. $V=\left(e^{\alpha t}-1\right)^{\frac{3}{\alpha}}$, here we consider $m=1$.

From equation (9) and (10), we obtain

$\frac{A}{B}=d_{1} \exp \left(\beta \int a^{-3} d t\right)$.

Here $\beta$ and $d_{1}$ are positive constants of integration. Considering $m=1$ and using equation (21) and (23), the metric potential can be obtained as

$$
\begin{aligned}
& A=d\left(e^{\alpha t}-1\right)^{\frac{1}{\alpha}} \exp \left\{\begin{array}{l}
-\frac{2 \beta}{9}\left(1-e^{-\alpha t}\right)^{3 / \alpha}\left(e^{\alpha t}-1\right)^{\frac{-3}{\alpha}}{ }^{2} \\
F_{1}\left(\frac{3}{\alpha}, \frac{3}{\alpha} ; \frac{\alpha+3}{\alpha} ; e^{-\alpha t}\right)
\end{array}\right\}, \\
& B=k\left(e^{\alpha t}-1\right)^{\frac{1}{\alpha}} \exp \left\{\begin{array}{l}
\frac{\beta}{9}\left(1-e^{-\alpha t}\right)^{3 / \alpha}\left(e^{\alpha t}-1\right)^{\frac{-3}{\alpha}}{ }_{2} \\
F_{1}\left(\frac{3}{\alpha}, \frac{3}{\alpha} ; \frac{\alpha+3}{\alpha} ; e^{-\alpha t}\right)
\end{array}\right\},
\end{aligned}
$$

where ${ }_{2} F_{1}$ is hyper geometric function and $d=\left(d_{1}\right)^{2 / 3}, k=\left(d_{1}\right)^{-1 / 3}$ satisfying $d \cdot k^{2}=1$.

The mean Hubble parameter $H$ for LRS Bianchi type-I metric may given by

$$
H=\frac{1}{\left(1-e^{-\alpha t}\right)} \text {. }
$$

The anisotropy parameter of the expansion $(\Delta)$ is obtained as

$\Delta=\frac{2 \beta^{2}}{9} e^{-6 t}\left(1-e^{-\alpha t}\right)^{-2(3-\alpha) / \alpha}$.

The shear scalar $\sigma^{2}$ is found as

$\sigma^{2}=\frac{\beta^{2}}{3} e^{-6 t}\left(1-e^{-\alpha t}\right)^{\frac{-6}{\alpha}}$.

The expansion scalar $\theta$ is found to be

$\theta=3 H=3\left(1-e^{-\alpha t}\right)^{-1}$.

The energy density $\rho^{(m)}$ and pressure $p^{(m)}$ of perfect fluid are obtained as

$$
\begin{aligned}
& \rho^{(m)}=\mu\left(e^{\alpha t}-1\right)^{\frac{-6\left(1+\omega^{(m)}\right)}{\alpha\left(3-\alpha^{(m)}\right)},}, \\
& p^{(m)}=\omega^{(m)} \mu\left(e^{\alpha t}-1\right)^{\frac{-6\left(1+\omega^{(m)}\right.}{\alpha\left(3-\alpha^{(m)}\right)}},
\end{aligned}
$$

where $\mu$ is positive constant of integration.

From equation (11), the energy density of dark energy component is obtained as

$$
\rho^{(\alpha e)}=\left(\frac{\lambda}{8 \pi+\lambda}\right)\left\{\begin{array}{l}
3\left(1-e^{-\alpha t}\right)^{-2}-\frac{\beta^{2}}{3}\left(e^{\alpha t}-1\right)^{-6 / \alpha} \\
-\left(\frac{16 \pi \omega^{(m)}+\lambda\left(3-\omega^{(m)}\right.}{2 \lambda}\right) \mu\left(e^{\alpha t}-1\right)^{\frac{-6\left(1+\omega^{(m)}\right)}{\alpha\left(3-\omega^{(m)}\right)}}
\end{array}\right\} .
$$

The EoS parameter of dark energy is as fallows.

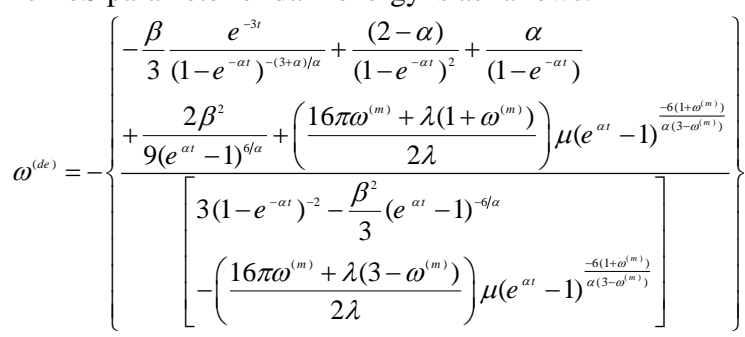

The deceleration parameter $q$ is the measure of the cosmic accelerated expansion of the universe. The behavior of the universe models is determined by the sign of $q$. The positive value of deceleration parameter suggests a decelerating model while the negative value indicates inflation. The deceleration parameter is given by and found to be

$q=\frac{d}{d t}\left(\frac{1}{H}\right)-1=\frac{\alpha}{e^{\alpha t}}-1$.

\section{Discussion}

The asymptotic behavior of the parameters of presented model is as fallows.

\begin{tabular}{lll}
\hline Parameters & $t \rightarrow 0$ & $t \rightarrow \infty$ \\
\hline$A, B, a$ & 0 & $\infty$ \\
$\rho^{(m)}$ & $\infty$ & 0 \\
$\rho^{(d e)}$ & $\infty$ & $\left(\frac{3 \lambda}{8 \pi+\lambda}\right)>0$ \\
& & $-\frac{2}{3}$ \\
$\omega^{(d e)}$ & Indeterminate & -1 \\
$q$ & $\alpha-1$ & 0 \\
$\Delta$ & $\infty$ & 0 \\
$\sigma^{2}$ & $\infty$ & \\
\hline
\end{tabular}

i) From the table, we have observed that the spatial volume $V$ and the directional scale factors $A$ and $B$ vanishes as $t \rightarrow 0$. However, the volume scale factor increases with time showing the late time acceleration of the universe. The other parameters such as $\rho^{(m)}, H, H_{x}, H_{y}=H_{z}, \Delta$ and $\sigma^{2}$ diverge as $t \rightarrow 0$. Thus the model exhibits a singularity at $t=0$ [36]

ii) The evolution of deceleration parameter $q$ is as shown in figure (1). The figure (1) shows that the universe accelerates after an epoch of deceleration. For $\alpha=3 / 2$ the deceleration parameter $\mathrm{q}$ is in the range $-1 \leq q \leq 0.5$ as shown by shaded region which is consistent with the observations made by Riess et al. [1] and Perlmutter et al. [2] and the present day universe is undergoing accelerated expansion.

iii) The model has high anisotropy and shear in the beginning of the evolution of the universe. It is observed that the anisotropy decreases to zero very quickly as shown in figure (2). Hence, the model reaches to isotropy after some finite time which matches with the recent observations as the universe is isotropic at large scale. The models which reduces to accelerating isotropic (Flat FRW model) is considered as standard cosmological models according to observations of Type Ia supernovae (SNeIa) given by Riess et al. [1, 37], 
Perlmutter et al. [2], the SDSS data given by Eisenstein et al. [38] and the three year WMAP data given by Spergel [4].

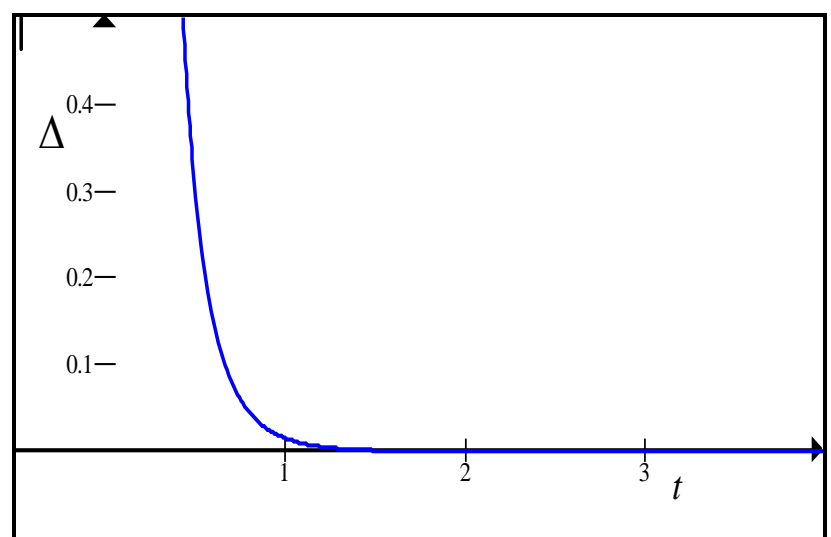

Fig. 2: Evolution of Anisotropy Parameter of Expansion $\Delta$ for $\alpha=1$.

iv) Here we observed that the universe exhibits the exponential expansion (also known as de-sitter expansion). Also, in this model at large time, $\omega^{(d e)}$ attains the value $\omega^{(d e)}=-\frac{2}{3}$, that is it converges to Quintessence $(-1<\omega<-1 / 3)$ region.

v) The available data set in cosmology, especially the SNeIa data [1, 2], the SDSS data [38] and the three years WMAP data [4] all indicate that the $\Lambda C D M$ model or the model that reduces near to $\Lambda C D M$ are serves as a standard cosmological model and is an excellent model to describe the cosmological evolution.

\section{Conclusion}

In this paper, LRS Bianchi type-I cosmological model in the context of $f(R, T)$ gravity theory have been studied. Here we discussed the model in which the solutions of the field equations are obtained for special form of deceleration parameter. It is observed that the model exhibits a initial singularity (Big-Bang) at $t=0$. The parameters such as $\theta, H, \rho^{(m)}, \rho^{(d e)}, \Delta$ and $\sigma^{2}$ diverge at the initial epoch. In this model the anisotropy of expansion dies out very quickly and attains isotropy after some finite time. It is also observed that the model asymptotically achieves a de Sitter phase and expands (accelerates) with the dominance of dark energy and the model matches the recent cosmological observations.

\section{Conflict of interests}

The authors declare that there is no conflict of interests regarding the publication of this paper.

\section{References}

[1] Riess A. G. et al.1998, Astron. J. 116,1009 [astro-ph/9805201] https://doi.org/10.1086/300499.

[2] Perlmutter S. et al.1999, Astrophys. J. 517565 [astroph/9812133].https://doi.org/10.1086/307221.

[3] Bennett C. L. et al. 2003, Astrophys. J. Suppl. 148,1 [astroph/0302207].https://doi.org/10.1086/377253.

[4] Spergel D. N. et. al. 2003, Astrophys. J. Suppl. 148, 175 [astroph/0302209].https://doi.org/10.1086/377226.

[5] Tegmark M. et al. 2004a, Phys. Rev. D 69 ,103501 [astroph/0310723].https://doi.org/10.1103/PhysRevD.69.103501.

[6] Tegmark M. et al. 2004b, Astrophys. J. 606, 702 [astroph/0310725].https://doi.org/10.1086/382125.

[7] Weinberg S. 1989, Rev. Mod. Phys. 61,.1

[8] Carroll S. M. 2001, Living Rev. Rel. 4,1 [astroph/0004075].https://doi.org/10.12942//rr-2001-1.
[9] Peebles P. J. E. and Ratra B.2003, Rev. Mod. Phys. 75, 559 [astroph/0207347].https://doi.org/10.1103/RevModPhys.75.559.

[10] Padmanabhan T. 2003, Phys. Rept. 380 ,235 [hepth/0212290].https://doi.org/10.1016/S0370-1573(03)00120-0.

[11] Carroll S.M., Duvvuri V., Trodden M., Turner M.S. 2004, Phys. Rev. D 70, 043528.https://doi.org/10.1103/PhysRevD.70.043528.

[12] Capozziello S., Nojiri S., Odintsov S.D., Troisi A. 2006, Phys. Lett. B 639, 135.https://doi.org/10.1016/j.physletb.2006.06.034

[13] Nojiri S., Odintsov S.D. 2006a, Physical Review D 74, 086005. https://doi.org/10.1103/PhysRevD.74.086005.

[14] Hendi S.H., Momeni D. 2011, Eur. Phys. J. C 71, 1823. https://doi.org/10.1140/epjc/s10052-011-1823-y.

[15] Sharif M., Arif S. 2012, Astrophys. Space Sci. 342, 237. https://doi.org/10.1007/s10509-012-1150-2.

[16] Nojiri S., Odintsov S.D. 2005, Phys. Lett. B 631, 1.https://doi.org/10.1016/j.physletb.2005.10.010.

[17] Nojiri S., Odintsov S.D.2011, Phys. Rep. 505, 59. https://doi.org/10.1016/j.physrep.2011.04.001.

[18] Cognola G., Gastaldi M., Zerbini S. 2008, Int. J. Theor. Phys. 47, 898.https://doi.org/10.1007/s10773-007-9516-x.

[19] Nojiri S., Odintsov S.D., Tretyakov P.V. 2008, Prog. Theor. Phys. Suppl. 172, 81.https://doi.org/10.1143/PTPS.172.81.

[20] Garcia N.M., Lobo F.S.N., Mimoso J.P. 2010, arXiv:1012.0953[gr$\mathrm{qc}]$.

[21] Bamba K., Capozziello S., Nojiri S., Odintsov S.D. 2012, Astrophys Space Sci 342, 155.

[22] Jamil M., Momeni D., Myrzakulov R. 2012a, Eur. Phys. J. C 72 , 2137.https://doi.org/10.1140/epjc/s10052-012-2137-4.

[23] Jamil M., Yesmakhanova K., Momeni D., Myrzakulov R. 2012b, Cent. Eur. J. Phys. 10(5), 1065.

[24] Harko T., Lobo F.S.N., Nojiri S., Odintsov S.D. 2011, Phys. Rev. D 84, 024020.https://doi.org/10.1103/PhysRevD.84.024020.

[25] Adhav K.S. 2012, Astrophys Space Sci. 339, 365.https://doi.org/10.1007/s10509-011-0963-8.

[26] Reddy D.R.K., Santikumar R., Naidu R.L.2012a, Astrophys. Space Sci. 342, 249.https://doi.org/10.1007/s10509-012-1158-7.

[27] Reddy D.R.K., Naidu R.L., Satyanarayana B. 2012b, Int. J. Theor. Phys. 51, 3222. https://doi.org/10.1007/s10773-012-1203-x.

[28] Chaubey R., Shukla A.K. 2013, Astrophys Space Sci 343, 415. https://doi.org/10.1007/s10509-012-1204-5.

[29] Samanta G.C. 2013, Int. J. Theor. Phys. 52, 2303. https://doi.org/10.1007/s10773-013-1513-7.

[30] Reddy D.R.K., Kumar R.S, Kumar T.V.P. 2013: Int. J. Theor. Phys. 52, 239.https://doi.org/10.1007/s10773-012-1325-1.

[31] Banerjee N., Das S. 2005, Gen. Relativ. Gravit 37, 1695. https://doi.org/10.1007/s10714-005-0152-6.

[32] Singha A., Debnath U. 2009, Int.J. Theor. Phys.48.

[33] Shamir M.F., Jhangeer A., Bhatti A.A. 2012, arXiv:1207.0708 [grqc].

[34] Poplawski N.J. 2006a, arXiv:gr-qc/0608031.

[35] Poplawski N.J.2006b, Class. Quant. Grav. 23, 2011. https://doi.org/10.1088/0264-9381/23/6/011.

[36] Bronnikov K.A., Chudaeva E.N., Shikin G.N. 2004, Class. Quantum Grav. 21, 3389.https://doi.org/10.1088/0264-9381/21/14/005.

[37] Riess A.G., et al. 2007, Astrophys. J. 659, 98 121.https://doi.org/10.1086/510378.

[38] Eisenstein D.J., et al. 2005, Astrophys. J. 633, 560.https://doi.org/10.1086/466512. 